\title{
Der meteorologische Einflufs auf Artenverbreitung und Rassenbildung bei den Insekten.
}

\section{Von Paul Meyer, Sušak (Kroatien) bei Fiume.}

Nachdem Prof. Dr. Heinrich Simroth, Leipzig, in seinem hochinteressanten Werke: „Die Pendulationstheorie" (Leipzig 1907, Konrad Grethlein) aus der R e ib is chschen Pendulationstheorie ${ }^{1}$ ) praktische und weitgehende Folgerungen für die Tiergeographie und Tierschöpfung gezogen und im grofsen und ganzen bisher auch mit entschiedenem Erfolge verfochten hat, ferner der gleiche Verfasser noch in einer weiteren Abhandlung: "Der Einflufs der letzten Sonnenfleckenperiode auf die Tierwelt" (Kosmos, Handweiser für Naturfreunde, Stuttgart, 1908, pag. 263-267) zahlreiche Tatsachen anführen konnte, welche die Abhängigkeit der irdischen Lebewelt von der wechselnden Sonnenwärme, bezw. die Parallele der meteorologischen und der biologischen Erscheinungen bestätigen, möchte ich an dieser Stelle ganz besonders auf die interessanten Beobachtungen hinweisen, welche sich seitens des sich sammelnd betätigenden Entomologen anstellen lassen und zu denen ein jeder sein Scherflein beitragen kann, wenn er sein Faunengebiet bezw. das Vorkommen und Auftreten der in seinem Exkursions-Terrain sich zeigenden, von ihm gesammelten Insektenformen genauer studiert, und zwar ganz ohne Rücksicht darauf, ob eine Art - je nach Seltenheit - für ihn von gröfserem oder von geringerem Werte ist.

Ein jeder Entomologe sollte sich für seine Exkursionen ein gewisses Vormerkbuch anlegen und darin nicht nur seine Fänge, sondern auch alle jene Arten anführen, die er, weil gemein, garnicht mit nach Hause gebracht hat. Solche Aufzeichnungen, zusammengefalst, werden sodann ergeben, dafs in dem einen Jahre diese oder jene Art an bestimmten Orten in grofsen Massen, in anderen Jahren nur sehr mäfsig oder fast garnicht vorgekommen ist, wie sich auch gewisse Beziehungen zu den Sonnenfleckenperioden nachweisen lassen werden, und zwar bei den Dipteren, Hymenopteren und Lepidopteren ${ }^{2}$ ) wahrscheinlich in die Augen springender als bei den Coleopteren, aber selbst bei letzteren werden sich ohne Zweifel mit der Zeit und bei regelmäfsiger, fleifsiger Beobachtung noch sehr viele interessante Tatsachen und Beziehungen finden lassen.

1) Paul Reibisch: Ein Gestaltungsprinzip der Erde, 27. Jahresber. Ver. f. Erdk. zu Dresden, 1901, pag. 105-124. - Ibid. 1905, pag. 39-52.

2) Es sei hier nur auf die sogenannten "Raupenjahre" hingewiesen, deren regelmälsige Begleiterscheinung in vielen Gegenden das üppige Auftreten von Calosoma-Arten ist. 
Unter Hinweis auf die über die Reibis ch-Sim rothsche Pendulationstheorie bereits von Dr. W. H o r $\mathrm{n}$ in dieser Zeitschrift 1908, pag. 288-289, 298-300 und 416-418, gemachten Ausführungen, sowie auf die Bemerkungen im "Kosmos" Stuttgart 1908, pag. 263, sei es mir gestattet - des besseren Zusammenhanges wegen - hier nochmals folgendes zu rekapitulieren. Vorausgeschickt sei noch, dafs jedenfalls die wahre Ursache der Pendulation uns heute noch unbekannt ist und möglicherweise, anstatt mit einem in Afrika aufgestürzten Monde, auch mit gewaltigen, periodischen Bewegungen im innersten Kerne unserer Erde etwas zu tun haben mag. Immerhin scheinen mir „Schwingungskreis" und "Kulminationskreis" biologisch die gedachte Bedeutung zu haben und somit wirklich auf eine Pendulation schliefsen zu lassen.

Nach Prof. Dr. Simroth hat also die Erde (gemäls Paul Reibischs Darstellung) aufser den beiden Rotationspolen, den Enden der Nord-Südachse, noch zwei Schwingpole: Ecuador und Sumatra, zwischen denen sie langsam hin- und herpendelt, so dafs die Erdachse nicht in gleicher Lage bleibt, sondern sich in bezeichneter Richtung verschiebt. Jede Pendelschwingung entspricht einer geologischen Periode. Der Ausschlag beträgt jedesmal 30-40 Grad, vielleicht auch weniger. Er ist am stärksten auf einem Meridian, der die Erde in eine westliche und östliche Halbkugel so zerlegt, dafs die Schwingpole Ecuador und Sumatra deren Mittelpunkte bilden. Dieser "Schwingungskreis" ist in Europa genau der $10^{\circ}$ ö. L. von Greenwich, der die Alpen in der oberen Rheinlinie in West- und Ostalpen scheidet, sowie in Deutschland die Städte Hamburg und Ulm durchläuft. Kamen wir im Diluvium in die zweite Eiszeitlage, so schwanken wir seitdem wieder nach Süden. In diesen rein mechanischen Verschiebungen, welche die Lebewesen fortwährend unter andere Sonnenstellung und anderes Klima bringen, ist nach Prof. Dr. Simroth das ganze Geheimnis der organischen Schöpfung, Artenbildung und Artenverbreitung $\mathrm{zu}$ suchen. Die Hauptumbildung erfolgte jedesmal bei polarer Schwingungsphase, wenn wir uns nach Norden bewegten. Europa, einschliefslich Nordafrika, aber ist der Herd, von dem die ganze Schöpfung ausgeht, und wo sie ihre Vollendung erreicht hat. Hier, unter dem Schwingungskreise, ist z. B. der erste Vogel als Archaeopteryx aufgetaucht und vermutlich wird man (nach Simroth) auch die Heimat des einzigen parasitischen Käfers: Platypsyllus castoris Rits. (am Biber) unter dem Schwingungskreise zu suchen haben: Rhône, Rhein, Elbe. Prof. Dr. Simroth hebt alsdann (l. c. pag. 145-146) hervor, dafs schon M a r s h 11 (längst vor der Entdeckung der Pendulation) eine ganze Reihe 
von Käfern anführe, die ein diskontinuierliches Areal bewohnen und wo die betreffenden Areale durch den Schwingungskreis scharf geschieden sind, so die verschiedenen Arten folgender Gattungen: Amphicoma Latr., Cylindromorphus Kiesw., Micrositus Muls., Penthicus Woll. (Opatroides Brull.), Oenas Latr., Lydus Latr., Amomphus Schönh., Vesperus Latr., Dorcadion Dalm, Deroplia Rosh. (Stenosoma Muls.) et Agapanthia Serv.

Bei der jetzigen äquatorialen Schwingungs-Phase, in der sich Europa bezw. unser atlantisch-indischer Nordquadrant befindet, wandert eine Anzahl Organismen sowohl von Osten wie von Westen unter den Schwingungskreis zurück. Sowohl diese Wanderungen wie eine besonders reiche Vermehrung hängen mit der ungefähr elfjährigen Sonnenfleckenperiode zusammen ${ }^{1}$ ). Die Wärmeperiode, in der wir uns gegenwärtig befinden, bewirkt ein besonders starkes Anschwellen dieser Erscheinungen. Tiere, die diesen Gesetzen folgen, sind auch in Form und Farbe sehr veränderlich, daher zur Bildung neuer Rassen geneigt und für die morphologische Forschung besonders wertvoll.

Gemäls diesen Schlufsfolgerungen bringt Prof. Dr. Simroth unter anderem die starke Vermehrung des Ulmenborkenkäfers (Eccoptogaster scolytus F.) in Belgien und in Nordfrankreich, in zehn- bis zwölfjährigen Perioden, auf die G. S e veri n aufmerksam gemacht hat ${ }^{2}$ ), in Beziehung mit den Sonnenfleckenperioden 1836, 1848, 1859, 1885 und 1896; ebenso die Eichenwickler-Kalamität in Schlesien in den Jahren 1888 und 1908, die Nonnen-Epidemien in verschiedenen Gegenden Deutschlands 1896-97 und 1907-08; dann die Heuschreckenplage 1907 in dem Pufstengebiete Hortobágy bei Debreczin in Ungarn und 1908 in Tunesien (gerade unter dem Schwingungskreis); endlich auch das Wespenjahr 1907 in Sachsen, welches nach ihm ein richtiges Wespenjahr war, das in der Schweiz ungewöhnlich viel Wespenbussarde erscheinen liefs und in welchem im Rheintal zahlreiche Starkästen von Wespen und Hornissen mit Beschlag belegt wurden.

Ein jeder Lepidopteren-Züchter wird schon Gelegenheit gehabt haben, sich von dem grofsen Einflufs zu überzeugen, welchen höhere Wärmegrade und direkte Einwirkung des Sonnenlichtes auf die Entwicklung der Falter haben.

1) Nach von Wolfer revidierten Wolfschen Untersuchungen der Sonnentätigkeit während der Jahre $1610-1874$ soll die Zeit vom Fleckenminimum zum Fleckenmaximum etwa 5 und diejenige vom Maximum zum Minimum etwa 6 Jahre betragen. Freilich kann sich das Maximum auch einmal selbst bis zu 2 Jahren verfrühen oder verzögern.

$\left.{ }^{2}\right)$ Bull. Soc. Centr. Forestière de Belg. 1906, pag. 401-404: Le scolyte de l'orme dans les plantations de la ville de Bruxelles. 
Nach Prof. Dr. Simroth (1. c. pag. 148) gehören hierher in erster Linie alle jene Versuche, die in der neueren Zeit der Entwicklungsphysiologie und Descendenztheorie von Weismann, Standfufs u. a. gemacht wurden.

Als ich mich in früheren Jahren in Hamburg noch mit Lepidopteren beschäftigte, zog ich Euchloë (Anthocharis) cardamines L. aus Eiern ${ }^{1}$ ) und konnte feststellen, dafs alle jene Imagines, deren Raupen und Puppen ich täglich mehrere Stunden starker und direkter Sonnenbelichtung ausgesetzt hatte, viel lebhafter gezeichnet waren, wobei ganz besonders das wesentlich feurigere Rotgelb und tiefe Schwarz der Vorderflügel bei den $\sigma^{\top} \sigma^{\top}$ auffiel. Solche Tiere sind dann natürlich auch in ihrem ganzen Wesen viel lebhafter, rastloser und flüchtiger als jene Imagines, von deren Raupen und Puppen das direkte Sonnenlicht stets fern gehalten wurde. Doch nicht nur bei Tagfaltern, sondern auch bei Bombyciden und Noctuiden erzielte ich das gleiche Resultat. Als besonders auffallend erwähne ich einen Versuch mit Acronycta alni L. Von dieser Spezies fand ich einmal eine Raupe auf einer kleinen Birke, welche ich auch mit Birkenlaub grofszog und ihr dann zwecks Verpuppung ein gröfseres Stück Torf anwies. Die Verpuppung erfolgte innerhalb des letzteren. Nach einiger Zeit brach ich vorsichtig das Torfstück auseinander, legte somit die Grube, in welcher die Puppe ruhte, frei, und setzte nun diese, nach vorheriger Befeuchtung des Torfes, des öfteren einige Zeit den Sonnenstrahlen aus, wodurch naturgemäls die Entwicklung des Falters beschleunigt wurde. Es schlüpfte alsdann ein ungemein lebhaftes $\sigma^{\top}$ aus, dessen Vorderflügel auffallend scharfe und tiefschwarze Zeichnung aufwies. Bei dieser Spezies scheinen auch jene Tiere, welche als Raupe mit Eichenblättern genährt werden, durchwegs dunkler auszufallen, als bei Fütterung mit Erlen- oder Birkenlaub.

Bei Pseudophia lunaris Schiff. ergibt solche Wärmebeeinflussung schöne, tiefbraune Bindezeichnung der Vorderflügel, während Imagines, deren Raupen und Puppen stets dunkel und kühl

1) Diese Eier sammelt man leicht auf feuchten Wiesen, wo sie sich einzeln an den Blüten- und Blattstielen auf Cardamine pratensis L. finden. Man pflückt diese Pflanzen möglichst langstielig, hält sie in Wasser, das oft zu erneuern ist, frisch und zieht auf diese leichte Weise die Raupen grofs, indem man jeweilig nach Bedarf noch neue Pflanzen hinzugibt, sowie die abgefressenen, von den Raupen verlassenen, entfernt. Als merkwürdig sei erwähnt, dafs ich bei nahezu erwachsenen Raupen beobachtet habe, dafs sie bei einem Mangel an frischen Futterpflanzen frische, noch weiche Puppen ausfressen, dafür dann aber selber weder gesunde, normal ausgebildete Puppen noch Imagines überhaupt ergeben. 
gehalten werden, blasse Färbung und weniger ausgeprägte Zeichnung zeigen. Bei der Nonne (Limantria [Psilura] monacha L.) läfst sich auf diese Weise, vielleicht besonders bei Fütterung mit Eichenlaub und speziell bei $\sigma^{\top} \sigma^{\top}$, die $a b$. eremita 0 . erzielen. Ähnliche und noch viel weitgehendere Experimente lassen sich mit Arctia caja L., wie auch mit Vanessa-Arten usw. anstellen, wenn man gleichextrem gefärbte und ausgebildete Exemplare beiderlei Geschlechtes sich begatten und dann deren Raupen und Puppen immer wieder unter der gleichen Wärme-, bezw. Kühlebeeinflussung sich mehrere Generationen hindurch entwickeln läfst.

Prof. Dr. Simroth bemerkt übrigens (1. c. pag. 148), dafs wohl alles, was bisher in dieser Richtung geleistet worden ist, sich auf Material und Vorkommnisse unter dem Schwingungskreise beziehe und es zweifelhaft erscheinen mag, ob die Versuche etwa in Amerika begonnen zu derselben Deutlichkeit geführt hätten. Es wäre daher von besonderem Interesse, die Pendulationstheorie auch an exotischem Material zu prüfen.

Sobald uns einmal die Biologie der verschiedenen Käferarten genauer bekannt sein wird, werden wir auch in dieser interessanten Ordnung Einflufs auf die Entwicklung einzelner Individuen nehmen können. So hat bereits $\mathrm{P}$ a uly ${ }^{1}$ ) "Zuchtversuche mit Tomicus typographus L. in künstlichem tropischen Klima" gemacht, und zwar in einem Warmhaus, in welchem die Victoria regia wächst, bei einer Temperatur zwischen $20^{\circ}$ und $40^{\circ}$ C., wobei sich ergab, dals die höhere Temperatur sowohl auf die Entwicklung selbst, als auch auf die Beschleunigung der Arbeit der aufserordentlich lebhaften Mutterkäfer von Einflufs ist.

Hier sei auch auf den interessanten Artikel von Dr. Curt He nning s, Karlsruhe, verwiesen ${ }^{2}$ ), welcher die Lebensgeschichte des Ips (Tomicus) typographus L. eingehend behandelt. Sehr bemerkenswert ist, dals auch nach diesem Berichte die „Wurmtrocknis", bezw. die übermäfsige Vermehrung des genannten Käfers, überall eine Begleiterscheinung der Sonnenflecken-Maxima war. Diese den Fichtenwaldungen verderblich werdende Krankheit wütete nämlich: in Ostpreufsen 1857-58, im Böhmerund Bayrischen Wald 1869-75, im Gouvernement Moskau 1882 - 83, in Baden (wie auch im Wienerwald) 1904-05. Dr. Hennings erwähnt, dafs häufig Raupen-, speziell Nonnenfrafs, welcher nicht selten ganze Fichtenbestände zum Absterben oder doch zum Kränkeln bringt, viel halbwelkes Holz liefere und dadurch eine Ursache der starken Vermehrung des Ips typographicus L.

1) Naturw. Ztschr. f. Land- und Forstwirtsch. 1906, pag. 160.

2 ) Ent. Bl. Schwabach 1908, pag. 67-73 und 92-97. 
sei. Hierdurch wird gleichzeitig bestätigt, dafs übermäfsiger Raupenfrafs ebenfalls gerade in den Jahren der "Wurmtrocknis", somit auch der Sonnenflecken-Maxima, beobachtet worden ist. Aulserdem bespricht Pfr. Wilh elm S chu st er (Ent. Bl. Schwabach 1908, pag. 146-147) die Überhandnahme von Afterraupen der Kiefernblattwespe Lophymus pini L. während der Jahre 1904 bis 1905 am Rhein, insbesondere im Gebiete des ehemaligen Mainzer Tertiärbeckens.

O t t o M e i $\int$ sner, Potsdam, schreibt (Ent. Bl. Schwabach 1908, pag. 143): „Wie auf manche Lepidopteren, so übt auch auf einige Coleopteren der Hunger eine die Entwicklung beschleunigende Wirkung aus. Besonders leicht läfst sich Adalia bipunctata L. zu vorzeitiger Verpuppung zwingen. Entzieht man einer Larve nach der letzten Häutung die Nahrung (und isoliert sie, denn sonst würde sie ihresgleichen fressen), so heftet sie sich nach einigen Tagen zur Verpuppung fest und gibt nach längerer Puppenruhe als gewöhnlich ein Zwergexemplar, und zwar wohl stets männlichen Geschlechtes." Derartige „Kummerformen" ergeben sich in der freien Natur anscheinend bei den Curculioniden besonders leicht, wobei natürlich dann solche Exemplare auch in der Punktur, Beschuppung und Beborstung von normalen Stücken wesentlich abweichen, was sehr oft zur Aufstellung neuer Arten verleitet haben mag. Übrigens kommen entgegengesetzt, gerade bei den Rüsselkäfer-Spezies, auch ganz abnorm grosse Stücke (Riesenformen) vor. (Nicht einsehen konnte ich: Zeitschr. f. wissensch. Ins.-Biologie 1908: A. C. J e n s e n, Silkeborg-Kopenhagen, „Über die Ursachen der Gröfsenunterschiede bei vielen Käfern".)

An dieser Stelle sei ferner der Sitzung der Deutsch. Ent. Ges. vom 16. XII. 1907 gedacht (vergl. D. E. Ztschr. 1907, pag. 285), gelegentlich welcher E. R e y Regenzeit- und Trockenzeit-Formen von Lepidopteren zeigte und dabei die Aufmerksamkeit auf etwaigen Saisondimorphismus bei Coleopteren lenkte, worauf dann Dr. W. H o rn als Beispiele des letzteren Frühformen und Spätformen bei Cicindelen anführte und auf Lixus und Hypera (Bestäubung der Frühform weifsgelb, der Spätform rot usw.) hinwies.

Aus dem Vorhergesagten erhellt, dafs, wenn die irdischen Organismen, neben der Pendulation, noch zufolge der Sonnenfleckenperioden bald ermälsigter, bald erhöhter Wärme ausgesetzt sind (wenn die Sonne durch ihre Bedeckung mit Flecken weniger leuchtet, so wird sie auch zur Zeit der Fleckenmaxima weniger wärmen, als zur Zeit der Fleckenminima), dieses von direktem Einflufs sowohl auf die Artenverbreitung als auch auf die Rassenbildung bei den Insekten sein mufs, und es lassen sich denn auch wirklich, wie bereits angedeutet, zahlreiche interessante Tatsachen 
feststellen, die dies zu bestätigen, bezw. hiermit im engsten $\mathrm{Zu}$ sammenhange $\mathrm{zu}$ stehen scheinen.

Nachfolgend führe ich einige Fälle an, welche auf Coleopteren Bezug haben und die teilweise schon den verschiedenen Sammlern selbst aufgefallen sind. Ich halte mich dabei an die meteorologische Bedeutung der Jahre und ziehe jeweilig die einer Sonnenfleckenperiode angehörenden, bezw. die einem Fleckenmaximum oder -Minimum zunächstliegenden Jahre zusammen.

Die Fauna meiner Vaterstadt Hamburg findet besondere Berücksichtigung, einerseits weil dieselbe über 2 Menschenalter fortlaufend gut erforscht und durch das von meinem Onkel zusammengestellte „Verzeichnis der in der Umgegend von Hamburg gefundenen Käfer", W. Koltze 1901¹), auch weiteren Kreisen bekannt ist, sowie andrerseits, weil ich selber in jenem, gerade unter dem Schwingungskreise liegenden Gebiete, jahrelang gesammelt habe.

Ich betone ausdrücklich, dafs ich mich bemühte, nur solche Spezies zu vermerken, deren Wiederverschwinden oder Seltenerwerden nicht etwa einfach damit begründet werden kann, dafs der Art - wie es innerhalb des Faunengebietes einer Grofsstadt ja leider fortwährend geschieht - die Lebensbedingungen entzogen wurden, indem etwa Nährpflanzen, alte Stämme, Pilzarten oder sonstige für die Entwicklung notwendige Bedürfnisse durch die Kultur ausgerottet worden sind.

Meiner Ansicht. nach dürfte vielmehr bei den angeführten Arten deren zeitweiliges Erscheinen oder üppigeres Auftreten auf meteorologische Beeinflussung zurückzuführen sein, und was speziell das Hamburger Faunengebiet anbelangt, so weisen dort die Einwanderungserscheinungen deutlich auf die bei Hamburg vorherrschenden Windrichtungen hin, nämlich auf Nordost und Südwest.

Im Hamburger Faunengebiete liefs auftreten

das Sonnenflecken-Maximum 1848:

Dictyoptera Aurora Herbst (in Schweden und Finnland heimisch),

das Sonnenflecken-Minimum 1856 :

Carabus variolosus F. (in Frankreich häufig),

das Flecken-Minimum 1867 :

Trechoblemus micros Herbst, Bembidion lunatum Duft., Stomis pumicatus Pz., Philonthus thermarum Aubé, Athous rhombeus Oliv., Prionocyphon serricornis Müll., Troglops cephalotes Oliv., Melandrya

1) Verh. des Vereins f. naturw. Unterhltg. in Hamburg, Bd. XI; Nachträge ebendaselbst Bd. XII (Dr. Hagedorn, 1904) und Bd. XIII (Dr. v. Sydow, 1907).

Deutsche Entomol. Zeitschrift 1909. Heft III. 
rufibarbis Schall. (alle Arten mehr dem südwestlichen Deutschland eigen),

das Flecken-M a x im u m 1870-71:

Bembidion humerale Strm., Pterostichus angustatus Dufts., Lathridius nodifer Westw. (alle 3 Spezies nordeuropäisch),

die Zeit von 1875 zum Flecken-Minimum 1878:

Cárabus variolosus F., Bembidion lunatum Dufts., Platynus micans Nic., Liodes nigrita Schmidt, Cyrtusa pauxilla Schmidt, Hydronomus alismatis Marsh. (auf Alisma plantago L.), Dryophthom corticalis Payk., Rhynchites Bacchus L., Chrysomela carnifex F. (alles Einwanderungen vom Südwesten),

das Sonnenflecken-Maximum 1882-83:

Hypera alternans Steph. (Finnland), Amara convexiuscula Mrsh., die Zeit von 1884 zum Flecken-Minimum 1889:

Pterostichus inaequalis Marsh., Dromius nigriventris Thoms., Cymindis vaporariorum L., Dadobia immersa Er., Velleius dilatatus F., Olophrum fuscum Grav., Geotrupes hypocrita Serv. $\left.{ }^{1}\right)$, Drilus concolor Ahrens, Rhipiphorus paradoxus L., Phyllobius sinuatus Fabr., Baris chlorizans Germ., Cryptocephalus ochroleucus Frm. (Arten der Lüneburger-, sowie Rheingegend),

\section{das Flecken-Maximum 1894-95:}

Tachys bisulcatus Nicol., Bradycellus distinctus Dej., Brachinus crepitans L. ${ }^{2}$ ), Hygrotus decoratus Gyll., Trichophya pilicornis Gyll. ${ }^{3}$ ),

1) Gemäls L. Bedel: Synonimies de Coléoptères paléarctiques, Abeille Vol. 30, No. 8 (1903), pag. 152, ist G. hypocrita Serv. (1825) synonym mit niger Marsh. (1802).

2) Nach W. Koltze (l. c. pag. 23) sind die am Elbstrand gesamımelten 2 Exemplare dieser bei Hamburg nicht eigentlich heimischen Art sicher angeschwemmt. Hier möchte ich auf den Bericht über die Verhandlungen der Naturforscher- und Ärzte-Versammlung 1907 verweisen, in welchem W. Krebs „Das meteorologische Jahr 1906-1907 Mitteleuropas mit besonderer Berücksichtigung der Hochwasser- und Sturm-Katastrophen " bespricht und deren Abhängigkeit von den Sonnenflecken betont. Die Verbreitung der Insekten durch Anschwemmen wird folglich ebenfalls von den Sonnenfleckenperioden beeinflufst und ist hierfür gewifs eine hübsche Bestätigung, dafs vom Brachynus crepitans L. das erste Hamburger Exemplar im Frühjahre 1863, somit gleichfalls kurz nach einem Flecken-Maximum, gefunden worden ist! Hätten nicht die besten Anschwemmplätze inzwischen längst der Kultur weichen müssen, so wäre vielleicht dieser Käfer auch während des besonders starken FleckenMaximums 1905-1906 wieder am Elbstrand gesammelt worden.

$\left.{ }^{3}\right)$ Diese zierliche, durch ihre haarfeinen Fühler sehr auffallende Art, siebte ich 1894 auf einer Elbinsel bei Hamburg aus dichten, modernden Schilflagen in sehr grofser Anzahl. In früheren Jahren ist die Spezies weder dort (wo alljährlich gesammelt wurde), noch sonstwo bei 
Medon castaneus Grav., Oxyporus maxillosus F.1), Tachinus elongatus Gyll., Arpedium quadrum Grav., Acidota cmentata Mannh., Atomaria atra Hbst., Lathridius nodifer Westw., Hypulus bifasciatus F., Otiorrhynchus laevigatus F., Phyllobius viridicollis F. (auf Spartium scoparium), Sitona puncticollis Steph., Rihynchites Mannerheimi Humm., Donacia fennica var. Malinowskyi Ahr. (alles Einwanderungen vom Nordosten),

das Flecken-Minimum 1900 :

Agabus nebulosus Forster, Rantus latitans Sharp, Atemeles pubicollis Bris., Nargus velox Spence, Sphaerites glabratus F., Corticeus

Hamburg gefunden worden. Dieses behende Tierchen dürfte auch nicht angeschwemmt gewesen sein, wie es bei vielen auf den Elbinseln gefundenen Insekten der Fall ist, vielmehr scheinen mir die ersten Brutkäfer dieser Art fliegend eingewandert gewesen zu sein. Speziell von den Staphylinen werden sich zum Zwecke des Wanderns wahrscheinlich viele Arten hoch in die Lüfte begeben, um sich dort vom Winde forttreiben $\mathrm{zu}$ lassen. Trichophya pilicornis Gyll. hätte sich vielleicht bei Hamburg länger gehalten, wenn nicht jene Elbinsel später in Hafenanlagen umgewandelt worden wäre. Mir ist unbekannt, ob sich die Art anderswo im Gebiete angesiedelt hat.

1) 1896 fand sich im Sachsenwalde (Friedrichsruh bei Hamburg) Oxyp. maxillosus F. in auf Buchenstümpfen wachsenden Pilzen (? Hypholoma fascicularis Huds.) nicht gerade selten. Aus einer solchen Pilzkolonie sammelte ich eine Reihe normal gefärbte Exemplare dieses hübschen Kurzflüglers nebst einem ganz schwarzen Stücke ( $a b$. Schönherri Mannh.), welcher interessante Käfer sich in coll. Koltze, Hamburg, befindet. Dieses Tier unterscheidet sich vom Oxyp. Mannerheimi Gyll. aus Schweden, Finnland, Livland usw. lediglich nur durch den sattelartigen Eindruck auf dem Halsschilde. Ich bin daher der Meinung, dafs auch Oxyp. Mannerheimi Gyll. keineswegs eine selbständige Art, sondern nur die ganz schwarze Form des Oxyp. rufus L. ist, dessen Bau und Halsschild sie besitzt und die wahrscheinlich im Norden häufiger auftritt. Hier spielt zweifellos klimatische Einwirkung eine Rolle, und es wäre interessant, genauer die Pilzarten festzustellen, in welchen sich Oxyporus entwickeln, wobei zu untersuchen ist, ob nicht etwa im Norden Oxyp. Mannerheimi Gyll. unter rufus L. vermischt lebt.

Was Oxyporus anbelangt, bin ich übrigens geneigt, anzunehmen, dafs gerade Mannerheimi Gyllh. und Schönherri Mannh. die älteren Formen sind (beide vielleicht aus einer gemeinsamen, schwarzen Urform der polaren Schwingungsphase unseres Quadranten hervorgegangen) und dafs sich aus ihnen erst später die Spezies rufus L. und maxillosus F. entwickelt haben.

In solchem Falle mag die Anlage für ganz schwarze Färbung im geheimen in den Geschlechtszellen der beiden letztgenannten Arten noch immer bestehen (durch eine lange Reihe von Generationen latent [verborgen] weitergegeben) und einzeln vorkommende Stücke von Schönherri Mannh. sind vielleicht als Rückschläge (Atavismen) aufzufassen (?). Züchtungsexperimente wären erwünscht!

Gewifs werden aber solche Rückschläge (Wiederhervorbringung von Vorfahrformen) in allererster Linie durch entsprechende meteorologische Beeinflussung zustande gebracht! 
linearis F., Cyllodes ater Hbst., Hoplia praticola Duft., philanthus Füssl., Chrysobothris affinis F., Pyropterus affinis Payk., Priobium castaneum F., Otiorrhynchus lugdunensis Boh. (nach W. Koltze, 1. c. pag. 126, wurde diese Art und ihre Larve 1901 in einer Gärtnerei den Syringen schädlich), Phytobius canaliculatus Boh., Ceuthorrhynchus atomus Boh., Rhynchaenus jota F., Gymnetron collinum Gyll., Apion aeneum F., Rhynchites Bacchus L., Anthribus nebulosus Forst., Caenoptera minor L., Plagionotus arcuatus L., Oberea oculata L. (Einwanderungen vom Südwesten),

das Flecken-Maximum 1905-06:

Dicranthus elegans F., vom Nordosten kommend und auf Equisetum palustre L. lebend.

Im Hamburger Faunen-Gebiete scheinen die folgenden Käferarten zur Rassenbildung meteorologischen Ursprungs zu neigen:

Acupalpus consputus Duft. (wesentlich heller als die süddeutschen Exemplare; bereits als Ac. Wimmeli Rttr. Wien. E. Ztg. 1893, pag. 44, beschrieben);

Deronectes 12-pustulatus F. (in Form und Zeichnung von französischen Stücken nicht unwesentlich abweichend);

Dermestes vulpinus F. (nach Dr. vo n Sy dow kommen Exemplare vor, bei denen in der Basalhälfte des die weifsen Flecke trennenden kahlen Mittelstreifens des fünften Abdominalsternits zahlreiche weifse Haare stehen, so dafs also diese Form fast die Zeichnung vom D. Frischi Kugel forma typica erreicht. Dieselbe Erscheinung erwähnt Ganglbauer Bd. IV, pag. 14, bei $D$. Frischi var. sibiricus Er.).

Die Insektenfauna der Stadt Ulm und deren Umgebung ist mir leider unbekannt, doch zweifle ich nicht, dafs auch dort durch die Sonnenflecken-Perioden bedingte Verschiebungen mehr oder weniger deutlich erkennbar sein werden.

Betrachten wir nämlich in Norditalien die Region des Schwingungskreises $\left(10^{\circ}\right.$ ö. L. von Greenwich), und zwar das Gebiet zwischen Po und Reno, welches mehr kontinentales Klima hat, so finden wir Ähnliches auch bei der Käfer-Fauna der Provinz Emilia, bezw. der Städte Piacenza, Parma, Modena und Bologna ${ }^{1}$ ).

1) Hier sei übrigens bemerkt, dafs sich natürlich derartige Verschiebungen nicht nur unter dem Schwingungskreis ergeben, sondern auch auf den anderen Gebieten. Am stärksten jedoch, und daher auch am leichtesten erkennbar, werden sie unter dem Schwingungskreis und nehmen dann kontinuierlich ab nach den Schwingpolen zu, wo sie gleich null sind. Unter dem Kulminationskreis aber stauen sich altertümliche Formen: er enthält die typische Reliktenfauna, die in den Schwingpolgebieten (Ecuador und Sumatra) am stärksten ist. 
Laut den "Note topografiche" von Prof. Andrea Fiori ${ }^{1}$ ) und Dr. Antonio $\mathrm{Porta}{ }^{2}$ ) zeitigte dort

das Sonnenflecken-Maximum 1894-95:

Acupalpus consputus Duft., Microglossa pulla Gyll. (in den Nestern von Lasius fuliginosus, brunneus und Formica rufa lebend), Atheta fluviatilis Kr., Gnypeta carbonaria Mannh., Oligota flavicormis Lac. (alles Spezies, welche mehr nordöstlich, in Südtirol (Trentino) usw. heimisch sind),

das Sonnenflecken-Minimum 1900 :

Harpalus rufus Brug., Amarochara forticornis Lac., Liogluta graminicola Grav., Homoeusa paradoxa Scriba (letztere Art in Nestern von Liometopum microcephalum lebend), Aloconota currax Kr., Myllaena gracilicornis Frm., Hypocyptus Pirazzolii Baudi, Quedius acuminatus Hochh., Staphylinus latebricola Grav., Othius melanocephalus Grav. et myrmecophilus Ksw., Vulda angusticollis Fv. ${ }^{3}$ ), Scopaeus rubidus Rey., Trogophloeus hirticollis Muls., Anthophagus abbreviatus F. (alles Arten, die mehr in südwestlich gelegenen Gegenden, Piemonte usw., vorkommen).

Nanche der vorgenannten Spezies werden durch die Flüsse Po und Reno in die Ebene geführt und daher auch unter Anspülicht gesammelt.

Dals aber besonders gerade Staphylinen zufolge meteorologischer Einwirkungen fliegend vom Nordosten gegen den Südwesten unter den Schwingungskreis zu wandern scheinen, zeigt sehr deutlich die Fauna der Insel Korsika. J. Sainte-Claire Deville führt nämlich in der Revue d'Entomologie 1906 (Catalogue critique des Coléoptères de la Corse, pag. 49-80) als für Korsika neu oder sehr selten u. a. folgende Spezies an: Stenus circularis Grav., cautus Er., Bledius cribricollis Heer, Trogophloeus fuliginosus Grav., gracilis Mannh., Thinobius longipennis Heer, minutissimus Fauv., deli-

1) Rivista Coleotterologica Italiana I, 1903, pag. 21, 24-35, 198-206.

2) Ibid., pag. 53-55, 98-102.

3) Prof. Fiori bemerkt, dafs er von dieser Art in jedem Herbste einige $\sigma^{\top} \sigma^{\top}$ in der Stadt Bologna und deren Umgebung an Mauern sammle, ohne bisher den richtigen Aufenthalt des Käfers bei Bologna entdeckt $\mathrm{zu}$ haben. Vielleicht hat dieses Tier seine Entwicklung überhaupt gar nicht in der Ebene und verläfst nur allherbstlich das Gebirge, um fliegend in die milderen Küstengegenden zu gelangen(?). Es dürfte sich dies wohl am besten an der toskanischen Küste feststellen lassen, weil Vulda angusticollis Fv. besonders in Toskana lebt. Vielleicht auch ziehen die Wärme erzeugenden Stadt-Ausdünstungen die Tiere an? So scheint bei Hamburg Acidota im Spätherbst in die Stadt zu fliegen, und bei Nachttieren z. B. ist erwiesen, dafs sie durch den Lichtreflex der Städte angezogen werden. 
catulus Kr., Lesteva Heeri Fauv., Acidota cmuentata Mannh., Porrhodites fenestralis Zett. (Teils Arten des hohen Nordens!)

A g o stin o D o de r o fù Giustino sammelte Xylodromus affinis Gerh. i. J. 1906 auf Sizilien (Ficuzza und Castelbuono) und führt in der Riv. Col. It. 1908, pag. 95, die Mannerheimia arctica Er., welche aus den Bergen Südtirols (Trentino) bereits bekannt ist, vom Gipfel der Majella ${ }^{1}$ ) in den Abruzzen auf, wo das Tier von A. Tirelli in einem Exemplare erbeutet wurde.

Nach Greiner (D. E. Ztschr. 1908, pag. 425) wurde auf Korsika, als für Europa neu, von E. Hopp: Axinotarsus pallitarsis Frm. gesammelt, welcher Fund ebenfalls sehr bemerkenswert ist.

Bei Fiume siebte ich in einem kleinen, gegen den Norden ganz abgeschlossenen, gegen den Süden (Meerseite) hingegen offenen Tale: Boreaphilus velox Heer. in einem Exemplare, welcher Käfer ziemlich über ganz Italien, die südliche Schweiz, Südfrankreich und Spanien verbreitet, meines Wissens aber bisher in ÖsterreichUngarn noch nicht gefunden worden ist. Diese Spezies wird mit dem Scirocco, einem feuchtwarmen, südwestlichen Winde von oft sehr beträchtlicher Stärke und Geschwindigkeit, herübergekommen sein, der uns in jedem Spätsommer und Herbst auch die Stechmücken oder Moskitos (Culex L. et Anopheles Meig.) vom Innern der Insel Cherso und wohl selbst aus Venedig, Ancona usw. beschert, letztere leider manchmal in unangenehm grofser Anzahl, zumal in den sogenannten „Mückenjahren", bei deren gelegent-

1) Im Monte Amaro $2795 \mathrm{~m}$ hoch.

Das Auftauchen nördlicher Formen weiter südlich und zwar speziell in der Region des Schwingungskreises und zu unserer Zeit, steht vollkommen im Einklange mit der Pendulationstheorie, indem nämlich gegenwärtig, wo sich unser Quadrant in äquatorialer Schwingungsphase befindet, die nördlicheren Gegenden desselben mit all ihren Organismen ganz allmählich immer mehr und mehr unter südliche Sonnenstellung gelangen, was unter dem Schwingungskreis eine bestimmte Rückwanderung nördlicher Formen gegen Süden zur Folge zu haben scheint. Bei der früheren polaren Schwingungsphase unseres Quadranten, während welcher dessen südlichere Breitengrade mit all ihren Lebewesen immer mehr und mehr unter nördliche Sonnenstellung gelangten, waren die Organismen ganz besonders bestrebt, sich von der Sonnenwärme loszumachen und ihre Verbreitung vom Schöpfungsherde im Mittelmeergebiete (unter dem Schwingungskreis) nach Norden auszudehnen. Fossile Funde aus der Tertiärzeit zeigen, dafs damals südliche Formen im nördlichen Europa vorkamen, die dann später zufolge der immer zunehmenden Kälte teils ausgestorben sind, teils sich umgebildet haben.

Stets war es aber der Schwingungskreis, wo die Organismen sich bunt durcheinander geschoben haben, wo die einen nach Osten, die andern nach Westen ausgewichen sind und wohin, bei äquatorialer Schwingungsphase unseres Quadranten (so gegenwärtig), sich die Arten unter Ausnützung der durch die Sonnenfleckenperioden bedingten Temperaturschwankungen zusammenzuziehen scheinen. 
lichem Zustandekommen meteorologische Faktoren gewifs eine grofse Rolle spielen ${ }^{1}$ ).

(Hier mache ich auf den interessanten Artikel von Fr. Regensberg: „Der Kampf gegen die Stechmücken“, Kosmos, Stuttgart, 1908, pag. 145-147 aufmerksam.)

Fliegend fing ich bei Fiume: Laemophloeus Krüperi Rttr., welche Art übrigens nicht nur aus Griechenland bekannt ist, wie irrtümlich im Cat. Col. Eur. 1906 angegeben und wie auch ich in dieser Zeitschrift 1907, pag. 186, fälschlich schrieb, sondern schon in dem "Verzeichnis der auf der dalmatinischen Insel Meleda vorkommenden Coleopteren" von L. Ganglbauer (Verh. d. k. k. zool. bot. Ges. Bd. 54, 1904, pag. 645) angeführt steht.

Die erhöhte Sonnentätigkeit der letzten besonders stark hervorgetretenen Periode 1905-1906 hat zweifellos sehr viele Verschiebungen des Verbreitungsgebietes mancher Insektenarten zur Folge gehabt.

Die Folgen der Maxima und Minima sind naturgemäls immer noch ein paar Jahre nachher zu verspüren ${ }^{2}$ ). So lassen die in den Jahren 1907-08 gemachten Funde noch recht deutlich den Zusammenhang mit dem Fleckenmaximum 1905-06 erkennen und ist $\mathrm{zu}$ erwarten, dafs selbst das Jahr 1909 (wenigstens in der Region des Schwingungskreises) noch derartige Formen zeitigen wird.

Als für die Käferfauna der Umgebung von Fiume auffallend konnte ich wahrnehmen, dafs beispielsweise Carabus catenatus Panz. und Nebria brevicollis F. in diesen Jahren an bestimmten Stellen viel häufiger auftraten als ebendaselbst zur Zeit des Fleckenminimums 1900, hingegen fehlte die damals nicht gerade seltene Nebria Dahli Strm. jetzt vollkommen, trotz unverändertem Terrain. Letztere Spezies scheint sich südlich verzogen zu haben. Theodor von Wanka, Teschen, sammelte dieselbe im Jahre 1906 in Unzahl in höheren Gebirgslagen der Herzegowina ${ }^{3}$ ), wo Genannter

1) Culex pipiens L. entwickelt sich übrigens im unteren Stadtteil Fiumes, in den Spülkanälen, woselbst (besonders in den letzten Jahren) auch Culex annulatus Schrk. bereits festen Fufs gefalst zu haben scheint. Die Tiere sind während der Scirocco-Tage, bezw. -Nächte, immer ganz besonders lästig.

2) Einige Arten vermögen sich natürlich neue Gebiete manchmal auch für ständig oder doch lange Zeit $\mathrm{zu}$ sichern. So findet man bei Hamburg Lathridius nodifer Westw., welcher Käfer dort zur Zeit des Fleckenmaximums 1894 - 95 sehr üppig auftrat, seitdem alljährlich regelmälsig und nahezu überall. Nach Koltze hat sich genannte Spezies während der 90er Jahre auch in anderen Teilen Deutschlands rasch verbreitet.

3) Coleopterologische Ergebnisse einer Reise in die Herzegowina, Ent. B1. Schwabach 1908, pag. 167-171, 188-194, 209-214 u. 228-233. 
damals auch Bembidion combustum Mén. ${ }^{1}$ ), ein Exemplar des Carabus montivagus Pall., sowie folgende Arten erbeutete: Euryusa castanoptera Kr. (in verhältnismälsig grofser Anzahl), Trichophya piliconnis Gyllh., Philonthus Bodemeyeri Epp. ${ }^{2}$ ), rufimanus Er., juvenilis Peyron, Pseudopsis sulcata Newm., Liodes carpathica Gglb., Cantharis longicollis Ksw. et sudetica Letzn., Liparus transsylvanicus Petri, alles Funde, welche die Wanderung östlicher Formen gen Westen, gegen den Schwingungskreis, bestätigen!

Mit Genehmigung des Herrn Victor Stiller, Zagreb (Agram), führe ich hier auch dessen Beobachtungen an, wonach an bestimmten Stellen des kroatischen Karstgebietes Carabus violaceus L. (? v. Germari Strm.) um 1900 herum (also zur Zeit des Sonnenflecken-Minimums) üppig vorkam, während sich der Käfer in den letzten Jahren (Zeit des Flecken-Maximums) garnicht zeigte. Andrerseits traten die Arten: Carabus Creutzeri F. (var.?) und Rosalia alpina L. in den Jahren 1905-07 (Flecken-Maximum) stellenweise üppig auf, fehlten aber um 1900 herum und was

1) Diese Spezies wird von Viktor Apfelbeck auch aus der albanesischen Landschaft Merdita angeführt (Koleopterol. Ergebn. der mit Subv. d. kais. Akad. der Wissensch. in Wien im Frühjahre 1905 nach Montenegro und Albanien ausgeführten Forschungsreise: Sitzungsber. d. kais. Akad. d. Wissensch. Wien 1907, Bd. CXVI, Abt. 1, pag. 493-506.)

Aufser dieser Art hat die Merdita u. a. auch die nachfolgenden Käfer mit dem Kaukasus gemein: Cicindela Fischeri Ad., Bembidion substriatum Chd., Chlaenius flavipes Mén., Laena merditana Apf. (prope piligera Wse.), Bergrothiella albanica Apf. (als Vertreter von Asclera Reitteri Ganglb.).

Der l. c., pag. 500-501, von V. Apfelbeck beschriebene Faunencharakter des Merdita-Gebietes ist im übrigen eine klare Bestätigung der von Prof. Dr. Simroth für den ,adriatischen Winkel " angegebenen Eigentümlichkeit, wonach jene Gegenden eine Menge altertümliche Lebewesen beherbergen (confer.: Höhlenfauna des Karstes!). V. A pfelbeck sagt mit Bezug auf Coleopteren: "Die Fauna der Merdita, welcher boreal-alpine Arten fehlen, hat typischen Reliktencharakter, der sich durch Auftreten zahlreicher Reliktendemiten und Arten mit diskontinuierlicher Verbreitung kennzeichnet. Aufserdem zeigt die Fauna Affinitäten nach verschiedenen Richtungen und läfst sich in folgende Elemente zerlegen: dalmatinische Arten (beziehungsweise Vertreter des Karstes), alpin-karpathische, pontische und bellenische Arten."

Da die besagte Forschungsreise Apfelbecks zur Zeit des letzten grolsen Sonnenfleckenmaximums unternommen wurde, würde es von besonderem Wert sein, die Käferfauna von Montenegro und Albanien einmal zur Zeit eines Sonnenfleckenminimums zu untersuchen. (Das nächste Fleckenminimum soll ungefähr im Jahre 1912 stattfinden.) Sicherlich werden sich dann auch dort manche interessante Verschiebungen erkennen lassen!

2) Nach J. Sainte-Claire Deville, Abeille (Vol. 30), 1906, N. 13, pag. 261-268: Contributions à la Faune française (Coléoptères), ist $P h$. Bodemeyeri Epp. = coerulescens Lac. 
speziell Ros. alpina L. anbelangt, so soll dieser Bock auch zur Zeit des Flecken-Maximums 1894-96 an denselben Plätzen häufig vorgekommen sein.

Des Weiteren bringe ich nachfolgende Mitteilungen in $\mathrm{Zu}$ sammenhang mit dem letzten Sonnenflecken-Maximum:

In der Ztschr. d. Naturw. Vereins in Posen, 1905 (Entomologie) pag. 28-29, berichtet E. S c h u h m a n n über das häufige Vorkommen des Schädlings Rhamnusium bicolor Schrk. in der Umgebung von Posen. Er fand den Käfer dort auf verschiedenen Laubhölzern brütend, insbesondere auf Ulmen, Eschen, Weiden, Pappeln und Rofskastanien.

$\mathrm{R} \mathrm{ud} \mathrm{olf} \mathrm{Tréd}$ l, Prüfening, fand den viele Jahre geradezu verschollen gewesenen Cryphalus intermedius Ferr. 1905 auf der Saiser-Alpe in Südtirol in ca. 1200-1400 m Meereshöhe (am Nordabhang, daher kühle, feuchte Lage) auf vom Schnee und Wind geworfenen Lärchen (Larix europaea Dec.) und bemerkt (Ent. Bl. Schwabach 1908, pag. 30-31) dazu: „in den letzten Jahren wurde der Käfer auch in Ober-Österreich (coll. Petz), in der Schweiz und in Kärnten (Dr. Gilb. Fuchs: Die Borkenkäfer Kärntens und der angrenzenden Gebirge: Naturw. Ztschr. f. Landund Forstwirtsch. 1905, pag. 232) auf Lärchen brütend angetroffen".

Nach V. T o r ka (Ent. Bl. Schwabach 1908, pag. 10 und 28) trat Cryptorrhynchus lapathi L. in den Jahren 1904 und 1906 (zufolge zweijähriger Generation) in der Niederung der Paklitz, einem Zuflusse der Obra an der Grenze der Provinzen Posen und Brandenburg, wo sein Brutbaum die Schwarzerle ist, sehr häufig auf.

Prof. J. R o u ba l, Prag, schreibt 1. c. pag. 13, dafs der sonst seltene Anoncodes ustulata F. in manchen Jahren sehr häufig und manchmal selbst in Städten vorkomme; so fing der Genannte am 21. Juli 1906 in München 7 Exemplare im Fluge. Derselbe Sammler fand 1907 von Eccoptogaster laevis Chap. Stämme der Schwarzerle (Alnus glutinosa L.) derart dicht besetzt, dafs durch den Larvenfrafs die Rinde völlig unterwühlt und das sonst regelmäfsige Frafsbild ganz verwischt war (1. c. pag. 12).

Vom Prionychus ater F. soll nach K. D o r n (l. c. pag. 49) bei Leipzig, wo sich der Käfer hauptsächlich im Mulm von Eichen and Kirschbäumen entwickelt, die Larve mitunter sehr häufig vorkommen (in welchen Jahren?). So fand Genannter in einem einzigen Kirschbaume einmal gegen hundert Larven in den verschiedensten Gröfsenstadien.

Robert Heinemann, Braunschweig, berichtet (Wanderungen von Käfern, Ent. Bl. Schwabach 1908, pag. 79-81), dafs sich bei Braunschweig der südeuropäische Hydroporus cana- 
liculatus Lac. eingebürgert habe und sich dort seit Jahren in grofsen Mengen in der Oker finde, ferner Phloeosinus thujae Perr., für den R. Tréd l von norddeutschen Gebieten Pommern und Posen angibt und der sonst nur in Süddeutschland zu finden ist, nördlich von Braunschweig in den Gebieten der Lüneburger Heide, um Gifhorn herum, aufserordentlich häufig sei und er im Sommer 1907 viele Büsche von Juniperus communis L. mit diesem Käfer besetzt gefunden habe. Endlich, dafs auch Leistus rufomarginatus Duft. ${ }^{1}$ ) und Pityophthorus Lichtensteini Ratzeb. bei Braunschweig eingewandert seien und während der Jahre 1904-07 sich ungemein häufig zeigten. Interessante Notizen über die Lebensweise von Pityophthorus Lichtensteini Ratzeb. gab Gerichtsassessor G e r ha rd: Ent. Bl. Schwabach 1908, pag. 157-162.

Nach Otto Meifsner, Potsdam (1. c. pag. 180, entdeckte Prof. Wanach kürzlich in Potsdam den bisher nur in Berlin, Marseille und Lenkoran gefundenen, nur etwa $1^{1 /} / 2 \mathrm{~mm}$ langen Käfer: Cartodere Schüppeli Rttr.

Sollte es nun wirklich reiner Zufall sein, dafs z. B. bei Hamburg die mehr dem nordöstlichen Europa eigenen Spezies besonders in den Jahren eines Sonnenflecken-Maximums, die mehr dem südwestlichen Deutschland eigenen Arten hingegen meistens immer in den Jahren eines Sonnenflecken-Minimums sich zeigen?

Ich glaube nicht! ${ }^{2}$ ) Dagegen ist begreiflich (zumal gelegent-

1) In den Ent. Bl. Schwabach 1908, pag. 101, teilt Dr. Ed. Everts, Haag, mit, dafs dieser Käfer an verschiedenen Plätzen Hollands, jedoch nur auf diluvialem Boden vorkomme und einmal in grofser Anzahl gefangen worden sei. Dies veranlafste mich, Genannten zu bitten, mir bekannt zu geben, wann solches der Fall war. Herr Dr. Everts schrieb mir darauf folgendes: "Leistus rufomarginatus Duft. ist von Herrn K. Kempers am 8. Juli 1894 (mit einem andern Herrn) in 57 Exemplaren bei Wageningen in der Provinz Gelderland gesammelt worden. Im übrigen ist die Art in Holland selten und wird nicht alle Jahre gesehen."

Es ist gewifs kein Zufall, dals auch dies üppigere Vorkommen in die Zeit eines Sonnenflecken-Maximums fällt! Interessant wäre es, festzustellen, in welchen Jahren L. rufomarginatus Duft. auf französischem Gebiete erbeutet worden ist.

$\left.{ }^{2}\right)$ Die schönen und geistreichen Worte Schillers: „Es gibt keinen Zufall und was uns blindes Ohngefähr nur dünkt, gerade das steigt aus den tiefsten Quellen", sind wohl für keine Sache zutreffender als für die Erscheinungen in der freien Natur. Meines Erachtens darf sie der Biologe bei Ziehung seiner Schlüsse getrost beherzigen, wenn er sich dabei nur genügend vor Augen hält, dals selbst in der freien Natur schon manches durch die Folgen der so vielfach widernatürlichen Kultur des Menschen in der natürlichen Entwicklung gestört und demzufolge entstellt, sowie schwer erkenntlich gestaltet wird. 
lich sehr grofse Gebiete von Sonnenflecken verdunkelt werden ${ }^{1}$ ), dafs ein merklicher Prozentsatz der gesamten Wärmestrahlung der Sonne durch die Flecke verloren geht ${ }^{2}$ ). Direkte Messungen der durch die Sonnenflecke hervorgebrachten Temperatur-Schwankungen auf der Erde lassen sich wegen der vielen lokalen Einflüsse auf die meteorologischen Verhältnisse nur sehr schwierig anstellen, doch scheinen selbe zu bestätigen, dafs die Gesamttemperatur der Erdatmosphäre vom Flecken-Minimum zum Maximum um etwa einen Grad schwankt. Bedenkt man nun, dals ausgerechnet worden ist, es bedürfe keiner gröfseren Temperaturerniedrigung als $3^{0}$ bis $5^{0}$, um jene Eiszeiten zu erklären, welche die Grenze des ewigen Schnees in unseren Alpenregionen um mehr als $1000 \mathrm{~m}$ herabdrückten und ganz Norddeutschland durch von Skandinavien sich herüberwälzende Gletscher unter einer mehrere hundert Meter dicken Eisdecke begruben ${ }^{3}$ ), so ist leicht verständlich, dafs die Sonnenflecken-Perioden auf die Verbreitung der einzelnen Insektenarten einen mehr oder weniger deutlich wahrnehmbaren Einflufs haben und Verschiebungen herbeiführen müssen, umsomehr als die Insekten zweifelsohne auf eine jede Temperaturschwankung durch Hervorbringung von mehr oder weniger wesentlichen Abweichungen, sei es in Gröfse, Farbe, Gesamthabitus oder aber in der Form einzelner Organe, ungemein viel leichter reagieren als höher entwickelte Organismen ${ }^{4}$ ). Ein Umstand, auf

1) So bedeckte eine Fleckengruppe, die im Februar und März 1905 selbst mit dem blofsen Auge sichtbar war, ${ }^{1 / 30}$ der uns zugewandten Sonnenhalbkugel, also ein ungefähr 200mal grölseres Gebiet als die ganze Erdoberfläche (vergl. Dr. M. Wilh. Meyer "Sonne und Sterne“, Stuttgart 1905, pag. 25 und 45 ).

2) Nach den Untersuchungen des verdienstvollen amerikanischen Sonnenforschers Langley strahlt von den Flecken nur $54 \%$ der Wärme aus, welche die übrige Sonnenoberfläche erzeugt.

3) Das Studium der Gletscher-Schwankungen hat ergeben, dafs ein Vorrücken der Gletscher in Perioden von 35 Jahren erfolgt, was wiederum mit den Sonnenflecken im Zusammenhange steht, indem nämlich auch in der Sonnentätigkeit dieselbe dreifach längere Periode hervortritt und immer jede dritte Fleckenperiode ganz besonders zahlreiche und grofse Flecke hervorbringt. So $1870-71$ und $1905-06$. Während dieser beiden Fleckenmaxima scheint z. B. bei Aschaffenburg der Oleanderschwärmer (Daphnis [Sphinx] nerii L.) gebrütet zu haben. Vergl. Dr. K. Flach: D. E. Z. 1907, pag. 14. Hier liegt wahrscheinlich der interessante Fall vor, dafs eine Art, welche zur Zeit der Fleckenminima zu weit nach Norden vorgedrungen ist (der Oleanderschwärmer fliegt bekanntlich selbst bis nach Norddeutschland), gelegentlich der Fleckenmaxima sich wieder südlicher verzogen hat.

$\left.{ }^{4}\right)$ Eine Ausnahme bilden einige absolut eurytherme (selbst gegen sehr grofse Temperaturschwankungen unempfindliche) Insekten-Spezies, deren Vorkommen (in unveränderter Form) sich, was speziell die paläarktische Region anbelangt, vom südwestlichen Portugal über das ganze 
Grund dessen sich in späterer Zeit wahrscheinlich zum grofsen Teile auch die verschiedenen Formen innerhalb der gleichen InsektenGattung erklären lassen werden, wie auch die grofsen Unterschiede, welche sich oft schon bei Tieren der gleichen Spezies aus differenten, durch den Schwingungskreis getrennten Provenienzen zeigen. Ich erinnere hier an die Verschiedenheit zwischen Exemplaren des Acalles pyrenaeus Boh. aus den östlichen Pyrenäen und solchen vom Riesengebirge, dann an Acalles roboris Curt. vom Nordseeküstengebiete und Tiere der gleichen Art von den südlichen $\mathrm{Ab}$ hängen des östlichen Alpengebietes usw. ${ }^{1}$ ).

Ohne Zweifel ist auch das vielfache Auftreten melanistischer Käferformen in ganz bestimmten Gegenden, ebenfalls zur Hauptsache eine Folge von meteorologischen Einwirkungen. Vielleicht

Gebiet hinweg, bis nach Ostsibirien, erstreckt. Dafs im übrigen die Käfer ein sehr feines Empfindungsvermögen für Luftdruckverhältnisse haben, bestätigen auch die interessanten "Notizen über Flugzeiten der Borkenkäfer" von Rudolf Trédl, Prüfening (Ent. Bl. Schwabach 1908, pag. 137-141). Ungeachtet einer gleichmäfsigen Temperatur von $+20^{\circ} \mathrm{C}$. schwärmten im geheizten Zimmer gezogene Pityogenes chalcographus L. und Pityophthorus micrographus L. erst dann, als die Lufttemperatur im Freien in den Mittagsstunden (der Flugzeit, $12-4 \mathrm{Uhr}$ ) $+11^{\circ} \mathrm{C}$. erreicht hatte, und unterbrachen ihr Schwärmen an kühleren, trüben Tagen.

(Hier sei noch auf Bachmetjews Schrift: „Verhalten der Insekten zur Temperatur" verwiesen, welche mir leider nicht vorliegt.)

1) Näheres über Rassenbildung bei den paläarktischen Acalles-Arten: Wien. Ent. Ztg. XXVII (1908), pag. 167-197.

Eine recht typische Bestätigung finden die Regeln der Pendulationstheorie in Ac. variegatus Boh. und dessen Rassen. Heimisch unter dem Schwingungskreise (Südfrankreich, Sardinien, Italien: Liguria, Emilia, toskanische Inseln, Umgebung Roms), lassen sich deutlich die Ausweichungen erkennen, nämlich:

a) gegen den Westpol: costatus Chevr. (Algerien), globulus m. (Marokko), angulipennis Woll. (Teneriffa);

a) gegen den Ostpol: Brisouti Rttr. (Griechenland), Pici Sol (Syrien), orientalis Sol. (Kaukasus), Paulmeyeri Rttr. (Turkestan).

Zufolge der äquatorialen Schwingungsphase unseres Quadranten strebt aber die Ostform Brisouti Rttr. unter den Schwingungskreis zurück und zeigt sich schon bei Rom, auf Sizilien usw., wogegen die forma typica des variegatus Boh. nach Osten vordringt und in Süddalmatien, der Herzegowina, Montenegro usw. auftaucht, wo sie mit Brisouti Rttr. zusammentrifft.

Ebenso bietet Omopliron im Mittelmeergebiet ein deutliches Beispiel: Unter dem Schwingungskreis findet sich auf Sardinien nur die Form sardoum Rttr. (Wien. Ent. Ztg. 1907, pag. 333), hingegen westlich (in Spanien) variegatum Oliv. und östlich (in Egypten) tessellatum Dej. Alle drei sind zweifelsohne Rassen der gleichen Art (vergl. auch Riv. Col. It. VI, 1908, pag. 19 et 209) und wohl aus Omophron limbatum F. hervorgegangen, dessen Vorkommen sich nach dem Katalog Bertolini über ganz Italien erstreckt. 
werden die schwarzen Formen daselbst besonders zur Zeit erhöhter Sonnentätigkeit (also der Sonnenflecken-Maxima) hervorgebracht und sind sämtlich Rückschläge (Atavismen)? Jedenfalls fand H. Bickhardt, Erfurt, im Juni 1908 auf Korsika 2 nene melanistische Formen ${ }^{1}$ ), welche von $\mathrm{O}$. Schneider in seiner, mir leider nicht vorliegenden Arbeit „Über Melanismus Korsischer Käfer " (Isis 1902, Heft 2, pag. 43-60) unter den aufgezählten 40 Spezies nicht genannt sind.

Die genauere Feststellung der Anzahl der Generationen bei jeder Käferart wird gleichfalls interessante Daten liefern. So gab bereits Ot to Mei $\int \mathrm{s} n$ er, Potsdam, in den Ent. Bl. (Schwabach) 1908, pag. 144, bekannt, dafs beispielsweise die Anzahl der Generationen von Chrysomela varians Schall. keine bestimmte ist und sich offenbar nach den meteorologischen Verhältnissen eines jeden Jahres richtet.

Endlich möchte ich diese kleine Arbeit nicht abschliefsen, ohne hier noch betont zu haben, dafs in manchen Fällen, wo wir glauben, auf Zuwanderung schliefsen zu müssen (indem plötzlich irgendeine Insektenform in einem Gebiete auftaucht, von wo dieselbe bisher nicht bekannt war), in Wahrheit die betreffende Form

1) Anthaxia millefolii $v$. Budtzi Bickh. und Cardiophorus Eleonorae $v$. humeralis Bickh. (Ent. Bl. Schwabach 1908, pag. 201-202).

Meifsners Melanismusarbeit in der Soc. Ent. Dez. 1907, sowie die Kontroverse zwischen Dr. Chr. Schröder und E. Fischer betreffs Entstehung des Melanismus (Ztschr. f. wissensch. Ins. Biolog. 1908, I und II) konnte ich bisher leider nicht einsehen.

Für die grofse Wahrscheinlichkeit, dafs wir von gewissen Käferarten die ältere Form (teilweise vielleicht noch aus der Zeit der polaren Schwingungsphase unseres Quadranten herrührend) in jenen schwarzen Rassen zu suchen haben, welche, bedingt durch entsprechende meteorologische Beeinflussung, in bestimmten Gegenden noch heute auftauchen, spricht auch folgender Umstand: Wie von Dr. K. Flach (D. E. Ztschr. 1907, pag. 11) ausgeführt, bezweckt ebenfalls bei den Insekten die schwarze Färbung lediglich, Wärme festzuhalten. Kälte und besonders Feuchtigkeit (Hochmoore) bewirken Schwarzfärbung. Während der Kälteperiode (zur Zeit polarer Schwingungsphase unseres Quadranten) wird also die Natur wohl auch die Käfer meistens schwarz hervorgebracht haben, damit sie die Kälte leichter überwinden. Tatsächlich zeigt die Fauna des hohen Nordens noch heute durchwegs dunkle Formen. Dals aber Staphylinen und Tenebrioniden, bei denen doch die schwarze Farbe vorherrschend ist, als ältere Käferformen anzusehen sind, wird wohl allgemein anerkannt.

Bei Korsika ergibt sich noch die interessante Parallelle, dafs die dortige Fauna neben melanistischen Käfern, auch nordische Staphylinen (also ebenfalls ältere Formen!) zeitigt.

Ein interessantes Kapitel bildet auch die an Nigrismen und Melanismen so reiche Insektenfauna von Turkestan. Hier scheinen sich eine Menge Formen erhalten und gestaut zu haben, die bei polarer Schwingungsphase unseres Quadranten nach Osten ausgewichen sind. 
an jenem Orte möglicherweise nur ( $\mathrm{zufolge}$ meteorologis $\mathrm{cher}$ Einwirkungen) entweder sich in gröfserer Anzahl entwickelt haben wird (indem sie auch früher schon vertreten war, jedoch so spärlich, dafs kein Sammler sie entdeckte), oder daselbst einfach aus anderen, bereits vorhanden gewesenen Formen, entstanden ist.

Sicherlich sind eben sehr viele der in unseren heutigen Katalogen als eigene, selbständige Arten figurierenden Insektenformen (von den in den letzten 3 Dezennien vom europäischen Territorium als nov. spec. beschriebenen Coleoperten wahrscheinlich sogar der gröfsere Teil!) de facto nichts weiter als Rassen wohlbekannter Spezies, zur Hauptsache bedingt durch klimatische Differenzen, Temperaturschwankungen usw. ${ }^{1}$ ).

1) Prof. Dr. Simroth schreibt (1. c. pag. 134) sehr richtig: „Leider ist bei den Kerbtieren die Artspalterei vielfach so weit gegangen, dafs man sich nicht nur auf rein morphologische, sondern auch auf geographische Tatsachen stützt und nicht ruht, bis man bei Formen, die weit voneinander getrennte Gebiete bewohnen, sonst aber in allem Wesentlichen übereinstimmen, feinste Differenzen findet und neue Spezies kreiren kann."

Es ist nicht zu leugnen, dafs vorläufig noch der bei weitem grölsere Teil der Entomologen der Ansicht huldigt, in den sich aus der modernen Auffassung der "Arten" ergebenden Resultaten und Schlufsfolgerungen nur „Phantasien“ erblicken zu dürfen. Die moderne Auffassung der "Arten" macht es sich zur Aufgabe, an der Hand biologischer Beobachtungen das eigentliche Wesen und den vollständigen Organismus der "Spezies" zu ergründen, anstatt nur die Charakteristik des einzelnen „Exemplares“ (als Imago) ins Auge zu fassen und allein diese zu beschreiben. Insolange einseitig das Letztere betrieben wird, bleibt uns das wirkliche Wesen der "Art" meistens überhaupt ganz unbekannt und wiederholen sich nur zusammenhangslose Neubeschreibungen untergeordneter Formen von bereits längst benannten Spezies, die dann in den späteren Katalogen einfach als synonym oder ganz nebensächlich anzuführen sein werden.

Die moderne Auffassung der "Arten" entspricht einer natürlichen Entwicklung, ist deshalb lebensfähig und wird ohne Zweifel dereinst auch ganz allgemein anerkannt werden müssen.

"Phantasie?", läfst Dr. Bruno Wille in seinen "Offenbarungen des Wacholderbaumes" (3. Aufl., 1907, Bd. I, pag. 290) den Professor Fechner, bitter lächelnd, sagen, „Sie meinen das offenbar geringschätzig - wie man die wundervolle Gabe, analogisch zu schlielisen und neue Zusammenhänge zu entdecken, leider in Gelehrtenkreisen zu behandeln pflegt. Phantasie ist fruchtbare Forschung, eine Art Experiment, ein Gedankenexperiment. Jede Weltanschauung ist Phantasie, ist eine philosophische Dichtung. Drum war ja auch so mancher Philosoph ein Dichter, so mancher Dichter ein Philosoph. Was hat denn Kepler zu einem der grölsten Entdecker gemacht? Seine Phantasie! Sie gab ihm ein, jene Gesetze zu vermuten, die hinterher durch Rechnen bestätigt wurden."

Dagegen gibt es nichts einzuwenden. Nehmen wir irgendwelche kulturelle Erfindung, mag sie noch so praktischer Natur und heute allgemeinverständlich, ja unentbehrlich sein, als Embryo galt sie der breiten Masse doch nur als "Phantasie"! 
Ein schönes Beispiel bietet auch Acalles punctaticollis Luc. Diese Art ist aus Algerien beschrieben und tritt in unveränderter Form auch in Tunis und Süditalien, somit in Gegenden mit einer durchschnittlichen Jahrestemperatur von $+17^{\circ}$ bis $18^{\circ} \mathrm{C}$. (Tunis $+19^{\circ}$ C.) auf. Aufserdem lebt aber die Art auch in Südfrankreich, Spanien und an der Westküste Norditaliens; alle Tiere jedoch aus diesen Distrikten (mit einer Durchschnittstemperatur von $+15^{0}$ bis $16^{0}$ C.) zeigen eine stärkere, üppigere Punktur des Halsschildes und weichen auch sonst von der Nordafrikaform etwas ab. Für diese nördlichere Rasse des Ac. punctaticollis Luc. schlage ich den Namen meteoricus vor und verweise im übrigen auf A. e F. S olari, Ann. del Mus. Civ. di Storia Nat. di Genova, Ser. 3, Vol. III (XLIII), 1907, pag. 503-504, welche Autoren bereits selber anführen, dafs die von ihnen var., italicus benannte süditalienische Rasse mit der Nominatform des Ac. punctaticollis Luc. aus Algerien identisch ist. In Andalusien wird wahrscheinlich Nordafrikaform leben, weil dort ebenfalls die höhere Durchschnittstemperatur herrscht.

Auch die diversen Rassen der Strophosomus-Arten: mufipes Steph., faber Hbst., retusus Marsh., sagitta Seidl. usw., werden vielleicht ihre Erklärung teils in der klimatischen Verschiedenheit der einzelnen Gebiete, teils in den Temperaturschwankungen der einzelnen Jahre finden. (Best. Tab. LXII, Genus Strophosomus Steph. Dr. K. Flach: Verh. des naturf. Verein. Brünn, XLV. Bd., 1907, pag. 201-230).

Gleiche Ursachen wird ferner in Italien bei Calathus luctuosus Latr. (gallicus Frm.) das sprungweise Vorkommen der Aberrationen: sirentensis Frac., Pirazzolii Putz. (Fracassi Heyd.), Luigionii Leoni et glabricollis Ullr., haben. (Näheres hierüber: G. L e o n i, Riv. Col. It. 1908 , pag. 49-59), endlich auch das Auftreten folgender Käferrassen im kahlen, baumwuchsarmen Gebiete Kalabriens: Paganetti Flach D. E. Z. 1907, pag. 15 (von Harpalus aeneus F.), calabrus Flach ibid. pag. 15 et 17 (三? Rebeli Apfel., von Poecilus cupreus L.), Amorei Ganglb. Verh. k. k. zool. bot. Ges. Wien, Bd. 57, 1907, pag. 196 (von Pterostichus bicolor Arag.). Vergl. G. L e o n i, 1. c., pag. $124-127$.

Poecilus Rebeli et var. hellenicus Apfelb. sind in der „Käferfauna der Balkanhalbinsel", I. Bd., 1904, pag. 254, beschrieben und schrieb mir der Autor wörtlich folgendes: „Poecilus Rebeli m. ist eine eigene A rt und kommt von der Herzegowina an bis Griechenland mit Poecilus cupreus L. gemeinsam vor, jedoch nur in Sümpfen, nahe dem Meere. Er ist stets dunkel b la u oder schwärzlichblau, nie kupfrig und hat ganz andere Halsschild-Skulptur, auch eine, allerdings ziemlich subtile Penisdifferenz. Es sind auch 
noch andere Unterschiede, auf die ich mich momentan nicht besinne".

Wahrscheinlich handelt es sich hier dennoch nur um eine dunkle, ältere Rasse des Poecilus cupreus L., die, wie so manche ältere Insektenart, uns in Sumpfgegenden erhalten blieb. So beachte man das Vorkommen von Acylophorus glabricollis Lac. et Wagenschieberi Ksw. in den Moorgebieten der Mark Brandenburg. Speziell von solchen älteren Formen aber dürfte sich die Entwicklung zur Zeit der Sonnenflecken-Maxima immer ganz besonders günstig und üppig gestalten.

Es ist selbstredend, dafs diese Zusammenstellungen keinerlei Anspruch auf Vollständigkeit machen können, wurde doch bisher seitens der Entomologen den Verbreitungsursachen der gesammelten Insekten meistens garnicht weiter nachgeforscht und ist daher auch nur verhältnismäfsig wenig diesbezüglich Verwendbares publiziert worden. So wird z. B. noch genauer untersucht werden müssen, ob nicht etwa das Auftreten der forma alata bei diversen, sonst ungeflügelten Käferarten, z. B. Carabus granulatıs L. ${ }^{1}$ ) und clathratus L., sowie die interessante Tatsache, dafs zeitweilig Tiere von zwei verschiedenen Spezies in copula angetroffen werden ${ }^{2}$ ), eben-

$\left.{ }^{1}\right)$ Dr. K. Flach fing 1875 einen Car. granulatus fliegend bei der Trockenlegung des Fasanerieweihers in Aschaffenburg una soll nach ihm (Wien. Ent. Ztg. XXV (1906) pag. 230, ähnliches soll auch am Neusiedlersee beobachtet worden sein. Gleichzeitig ist aber granulatus von allen $\mathrm{Ca}$ raben die weitverbreitetste Art!

Werden nicht solche Ausnahme-Exemplare vielleicht nur die Wiederhervorbringung des Insektes in seiner älteren Form bedeuten? Derselbe Fall liegt möglicherweise auch bei denjenigen Käfern vor, welche - von gewöhnlich blind vorkommenden Arten - einzeln mit Augen auftreten (?). Dr. Flach hat für die Neuglenes-Arten das regelmäfsige Vorkommen einer grofsaugigen, geflügelten "Wanderform" nachgewiesen und führt an, daf's auch Ptiliolum Oedipus Flach eine sehende "Wanderform" habe.

Für Spezialisten dürfte es sich verlohnen, die hier angedeutete Atavismustheorie in den von ihnen bevorzugten Gruppen weiter zu verfolgen, möglichst an Hand von Züchtungs-Experimenten. Ich glaube die Theorie hat einen fruchtbaren Boden und wird mit der Zeit zu manchen Aufschlüssen führen. Auch für diesbezügliche Untersuchungen und Beobachtungen ist natürlich das Gebiet des "Schwingungskreises" am geeignetsten, weil eben dort die jüngsten Umformungen stattgefunden haben, so dafs in den Geschlechtszellen vieler der heute dort lebenden Arten gewifs noch manche Eigenschaften älterer Vorfahren latent vorhanden sind, welche bei entsprechender meteorologischer Beeinflussung bei einzelnen Individuen dann und wann wieder zur Entfaltung gelangen, ohne (wohlverstanden) sich dauernd halten zu können.

2) G. Coniglio Fanales (Il Naturalista Siciliano, An. XVIII, 1905-1906, pag. 220) beobachtete Psilothrix protensus Gené ơ mit Ps. nobilis Kiesw. O in copula. E. Ragusa erwähnt dazu, dals er Chrysomela viridana Küst. $\sigma^{\top}$ mit $C h r$. polita L. + , wie auch umgekehrt polita $\sigma^{\top}$ mit viridana $q$ des öfteren in Sizilien in copula angetroffen habe. 
falls $\mathrm{zu}$ den Sonnenflecken-Perioden in irgendwelcher Beziehung stehen, indem möglicherweise auch solche Fälle bestätigen, dafs dem Bestreben, sich auszubreiten oder aber neue Rassen und Arten entstehen zu lassen, auf Grund meteorologischen Einflusses, Vorschub geleistet wird.

Es sollte mich freuen, wenn meine vorliegende Studie wenigstens einem Teil der vielen sich sammelnd betätigenden Entomologen Veranlassung sein würde, fortan auch den meteorologischen Einwirkungen auf das von ihnen durchforschte Gebiet ein Augenmerk $\mathrm{zu}$ schenken und alle damit vermutlich im Zusammenhange stehenden Beobachtungen, Funde und Entdeckungen in dieser Zeitschrift oder an sonst geeigneter Stelle bekanntzugeben.

\section{Nachschrift.}

Kurz vor Absendung meines Manuskriptes kam mir durch Güte des Herrn Reg.-Rat Med. Dr. Ottokar Nickerl, Prag, noch dessen für das Studium der Mikrolepidopteren wichtige Arbeit zu: "Die Motten Böhmens (Tineen)" — Ges. für Physiokratie in Prag, 1908: Beiträge zur Insekten-Fauna Böhmens, VI - und auf meine briefliche Anfrage teilte mir der Genannte mit, dafs in Böhmen im Jahre 1861 Heliozela sericiella Hw., in den Jahren 1882-84 Depressaria Douglasella Stt. und im Jahre 1897 Yponomeuta malinellus Z $\mathrm{Z}$. in grofsen Mengen und daher teilweise schädlich aufgetreten sind. Auch dies scheinen somit Folgen der bezüglichen Sonnenflecken-Maxima gewesen zu sein, während sich Yponomeuta evonymellus L. während des Minimums 1900 in Böhmen in ungeheuren Mengen und verwüstend gezeigt hat.

Ohne im übrigen dieses interessante Gebiet meinerseits $\mathrm{zu}$ beherrschen, will ich doch nicht unterlassen, anzuführen, dafs unter den in der besagten Arbeit besonders vermerkten Funden, nachfolgende Spezies sich wie folgt gruppieren:

Anscheinend liefs in Böhmen auftreten

das Sonnenfle cken-M aximum 1859-60:

Xystophora unicolorella HS., Argyritis libertinella Z., Stathmopoda pedella L., Cyphophora idaei Z., Coriscium cuculipennellum Hb., Adela associatella Z.,

das F lecken-Minim um 1867 :

Swammerdamia alpicella HS., Bryotropha decrepidella HS., Psecadia funerella F., Depressaria petasitis Stdfs., Diplodoma marginepunctella Stph., Tinea fulvimitrella Sodof., parasitella $\mathrm{Hb}$., arcuatella Stt.,

Deutsche Entomol. Zeitschrift 1909. Heft III. 
das Flecken-Maximum $1870-71$ :

Gelechia muscosella Z., virgella Thb., Xystophora atrella Hw., Chrysoclista Linneella Cl., Stagmatophora serratella Tr., Lithocolletis spinolella Dup., salictella Z., Lyonetia pulverulentella Z.,

das Flecken-Minimum 1878 :

Depressaria nervosa Hw., Coleophora binotapennella Dup., Oinophila $V$-flavum Hw.,

das Flecken-Maximum 1882-83:

Sophronia Sicariella Z., Depressaria impurella Tr., olerella Z., Coleophora niveicostella Z., Nepticula Nickerli Rebel, argentipedella Z., Incurvaria flavimitrella $\mathrm{Hb}$.

Ich mufs es den Spezialisten überlassen, zu untersuchen, ob diese Gruppen bestimmte Einwanderungs-Richtungen erkennen lassen, wobei ich auch noch darauf hinweisen möchte, dafs nach Prof. Dr. Simroth sich einige der dem "adriatischen Winkel“ eignen, altertümlichen Lebewesen um den Ostflügel der Alpen herum und hinauf bis mindestens Böhmen, erhalten haben sollen. Findet etwa letzteres auch in der Insektenfauna Böhmens einige Bestätigungen? (Unter den Cerambyciden könnten hier vielleicht die Arten Leptura thoracica Creutz., Letzneria lineata Letzn. und Neoclytus erythrocephalus F. in Frage kommen.)

Ich versäume nicht, Herrn Reg.-Rat Med. Dr. Ottokar Nickerl, Prag, an dieser Stelle meinen ganz besonderen Dank dafür auszusprechen, dafs er die Güte hatte, mich auf seine interessanten Berichte aufmerksam zu machen über die in den Jahren 1875-1890 der Landwirtschaft Böhmens schädlichen Insekten ${ }^{1}$ ), durch welche sich der Genannte ein bleibendes Verdienst um die Feststellung des periodischen Massenauftretens verschiedener, für die böhmische Landwirtschaft schädlicher Insektenarten gesichert hat und welche $u$. a. folgendes zeigen:

In Böhmen liefs massenhaft und demzufolge schädlich auftreten

das Sonnenflecken-Maximum 1882-83:

Charaeas graminis L., Entomoscelis adonidis Pall., Gryllotalpa vulgaris L., Hylobius abietis L., Lophyrus similis Htg., Neuronia popularis F., Phyllobius oblongus L., Retinia Buoliana Schiff., Scolytus Ratzeburgii Jans., Sirex gigas L., Tetranychus telarius L. (sind nicht

1) Vergl. Gesamtübersicht im Berichte der im Jahre 1890 der Landwirtschaft Böhmens schädlichen Insekten (Prag 1891, Selbstverlag), pag. $1-19$. 
vielleicht manche dieser Insekten auch während der späteren Maxima, etwa um die Jahre 1894-95 und 1905-06 herum, üppig in Böhmen vorgekommen?),

die Zeit von 1875 zum Flecken-Minimum 1878, wie (charakteristischerweise) auch jene von 1885 zum Minimum 1889:

Agrotis segetum Schiff., Aporia crataegi L., Bombyx neustria L., Bothynoderes punctiventris Grm., Cassida nebulosa L., Cecidomyia brassicae Win., Eccoptogaster rugulosus Ratz., Cochyllis ambiguella Hb., Jassus sexnotatus Fall., Mamestra brassicae L., Melolontha vulgaris F., Otiorrhynchus ligustici L., Phytonomus polygoni F., Plusia gamma L. ${ }^{1}$ ), Porthesia chrysorrhoea L., Silpha opaca L. ${ }^{2}$ ), Thrips cerealium Hal. ${ }^{3}$ ), Zabrus gibbus F., Tanymecus palliatus F.

Endlich seien hier aus dem Tätigkeitsbericht der entomologischen Sektion der Gesellschaft für Physiokratie in Böhmen über die Zeit von 1903-08 noch folgende, das letzte Sonnenflecken-Maximum 1905-06 betreffende Vorkommnisse angeführt:

Im Jahre 1907 wurde aus dem Kaplitzer Bezirke im südlichen Böhmen das massenhafte Auftreten der Raupe eines Kleinschmetterlings (Plutella cruciferarum Z.) gemeldet, welche den Kohlfeldern durch Abfressen der Blätter Schaden zufügte. Als natürlicher Feind und Schmarotzer dieses Schädlings entwickelte sich aus dessen Puppen gleichzeitig eine Ichnermoniden-Art in sehr grofser Individuenzahl.

1905 trat die Getreidehalmfliege (Chlorops taeniopus Mg.) in der Egerer Gegend als empfindlicher Schädling auf.

1) Es ist bemerkenswert, dafs diese Eule in Böhmen das eine Mal (1871) während eines Sonnenflecken-Maximums, hingegen andere Male (z. B. 1865 und 1879) zur Zeit eiñes Flecken-Minimus massenhaft und schädlich auftrat. Dieser Umstand jedoch findet wahrscheinlich darin seine Erklärung, dafs Piusia gamma L. ein ausgesprochenes Wandertier ist. Der Schmetterling zieht zeitweilig in ganzen Scharen und in ganz bestimmter Richtung über Wiesen, Felder und Länder dahin. Angenommen also, die Spezies ist im Jahre 1871 von Nordost gen Südwest durch Böhmen gezogen, so wird sie 1879 (auf ihrem Rückzuge) wahrscheinlich gerade in der entgegengesetzten Richtung, nämlich von Südwest gen Nordost, Böhmen durchquert haben, wie denn auch Plusia gamma L. im Jahre 1879 durch seine ganz enorme Ausbreitung und Vermehrung in einem grofsen Teile Deutschlands und Frankreichs bedeutendes Aufsehen erregt hat. Als eifriger Verfolger der Raupe wurde ziemlich häufig Calosoma sericeum Fab. konstatiert, der sonst in der Prager Umgebung eine seltene Erscheinung war.

$\left.{ }^{2}\right)$ Die Larven dieses Käfers fressen die Keimpflänzchen der Zuckerrübe (Beta vulgaris L.) ab. Auch Atomaria linearis Steph. und Bothynoderes punctiventris Germ. treten zeitweilig in Böhmen als Rübenschädiger auf.

3) Dieses Insekt erwies sich auch 1903 wieder als Getreideschädling an Kornähren bei Königgrätz. 
Eine Wanzenart, Geocoris ater, wird nicht als Schädling, wohl aber als seltenes Insekt wegen ihres Vorkommens in Schlan verzeichnet.

Ferner wird auch eines andern, in Böhmen sonst ziemlich selten beobachteten, wanzenartigen Insekts, Podops inuncta, als faunistischer Notiz gedacht, welches im ersten Frühling 1908 an den Sommerlehnen des Moldauufers bei Troja durch Prof. Höhm mehrfach erbeutet wurde.

Zum Schlufs wird über eine bemerkenswerte Erscheinung berichtet, wonach am 28. Juli 1907, etwa um $1 / 210 \mathrm{Uhr}$ abends, in einem hellbeleuchteten Restaurationsgarten in Saaz ein Massenschwarm von vielen tausenden Individuen einer Laufkäferart, und zwar Harpalus pubescens Müll. (ruficornis F.) zugeflogen kam und sich überall niederliefs, so dafs in einigen Augenblicken die Kleider der dort anwesenden Gäste, Tische, Gläser und der Erdboden, kurz alles von den Tieren bedeckt war und die anwesende Gesellschaft den Garten verlassen mufste.

\section{Notiz über das Vorkommen zweier grofser Insekten in Süddeutschland.}

Von Dr. W. von Reichenau, Mainz.

1. Scolia haemorrhoidalis in Bayern.

Das fortgesetzte Fehlen dieses grofsen Hymenopters in unsern deutschen Listen veranlafst mich endlich, eine Beobachtung über das unzweifelhafte Vorkommen der "Dolchwespe", wie T a s chenberg sie (Brehms Tierleben, Insekten) nennt, in Deutschland zu veröffentlichen.

Im Nachsommer des Jahres 1872 befand ich mich an einem schönen Nachmittage am Rande eines Tannenwaldes in Südexposition auf meinem damaligen Anwesen Hof Litzelau bei Miesbach in den oberbayrischen Voralpen. In einiger Entfernung vom geschlossenen Bestande war eine Hecke mit vielen einzelnen Tannen, Bergahorn und Eichen nebst Baumstubben, die etwa fufshoch über dem Boden abgesägt waren. Ohne Netz und Gewehr war ich ausgegangen, lediglich um nach dem Weidevieh zu schauen, welches sich zwischen dem Walde und der Einzäunung tummelte. Ich stand ruhig. Plötzlich wurde ich von einem lauten Surren erschreckt, ich sage, der Tatsache entsprechend, erschreckt, denn bei der fast absoluten Stille, die hier und da nur einmal von 


\section{$2 \mathrm{BHL}$ Biodiversity Heritage Library}

Meyer, Paul. 1909. "Der meteorologische Einflufs auf Artenverbreitung und Rassenbildung bei den Insekten." Deutsche entomologische Zeitschrift 1909(3), 381-406. https://doi.org/10.1002/mmnd.48019090305.

View This Item Online: https://www.biodiversitylibrary.org/item/103377

DOI: https://doi.org/10.1002/mmnd.48019090305

Permalink: https://www.biodiversitylibrary.org/partpdf/235957

\section{Holding Institution}

Harvard University, Museum of Comparative Zoology, Ernst Mayr Library

\section{Sponsored by}

Biodiversity Heritage Library

\section{Copyright \& Reuse}

Copyright Status: Public domain. The BHL considers that this work is no longer under copyright protection.

This document was created from content at the Biodiversity Heritage Library, the world's largest open access digital library for biodiversity literature and archives. Visit BHL at https://www.biodiversitylibrary.org. 